\title{
Ultra-Thin Artificial Magnetic Conductor for Gain-Enhancement of Antenna-on-Chip
}

\author{
Yiyang Yu, Student member, IEEE, Zubair Akhter, and Atif Shamim, Senior Member, IEEE
}

\begin{abstract}
System-on-Chip (SoC) has become an attractive solution to achieve highly integrated wireless systems. However, the Antenna-on-Chip (AoC) suffers from poor radiation due to the lossy silicon substrate in standard CMOS processes. Artificial Magnetic Conductors (AMC) with the ground plane above the silicon can enhance the radiation, however, fitting the AMC completely in the thin stack-up $(\sim 10-15 \mu \mathrm{m})$ is extremely challenging, particularly for frequencies below $100 \mathrm{GHz}$. In this paper, Metallic Posts (MP) and Embedded Guiding Structures (EGS) have been investigated to reduce the AMC thickness by employing the available vias and metal layers in the stack-up. An in-house CMOS-compatible process has been used to realize the AoC, where typical low-conductivity adhesion layers have been avoided to reduce the undesired losses by using the surface roughness in a unique fashion. With MP and EGS approaches, AMC thickness can be reduced by $33 \%$ and $41 \%$ respectively. The AMC with EGS fits within an oxide of a thickness of $16 \mu \mathrm{m}$. A monopole antenna, backed by this AMC, demonstrates a gain of $5.85 \mathrm{dBi}$ and radiation efficiency of $57 \%$ at $94 \mathrm{GHz}$, bettering the gain and radiation efficiency by $9.15 \mathrm{~dB}$ and $42 \%$ respectively as compared to the case without AMC.
\end{abstract}

Index Terms - Artificial magnetic conductor, embedded guiding structure, gain enhancement, metallic posts, on-chip antenna, system-on-chip.

\section{INTRODUCTION}

$\mathrm{W}$ IRELESS System-on-Chip (SoC), where all functional modules are integrated on a single chip, has attracted considerable research interest with the advantages of high-level integration, low power consumption, and low cost [1-4]. Typically, antenna is the largest part of a wireless system, and previously it was not feasible to integrate it on a chip. However, due to the push towards millimeter-wave spectrum, antenna sizes have dropped to the order of millimeters and thus become compatible with the typical chip dimensions [4-6]. On the other hand, silicon $(\mathrm{Si})$ based semiconductor technologies, such as a Complementary Metal Oxide Semiconductor (CMOS) process, have come a long way and thus high-frequency circuits and antennas can be realized on a single chip in a compact fashion [7].

Though, the size of the mm-wave Antenna-on-Chip (AoC)

This paragraph of the first footnote will contain the date on which you submitted your paper for review. It will also contain support information, including sponsor and financial support acknowledgment. For example, "This work was supported in part by the U.S. Department of Commerce under Grant BS123456".

The authors are with King Abdullah University of Science and Technology, Thuwal, 23955 KSA (e-mail: yiyang.yu@ kaust.edu.sa). has become compatible with the typical CMOS chip dimensions, however, the CMOS stack-up is still not favorable for AoC implementation. This is mainly because of the very conductive Si substrate that also has a very high permittivity. Further, the embedded metal layers in a very thin silicon dioxide $\left(\mathrm{SiO}_{2}\right)(\sim 10-15 \mu \mathrm{m})$, as shown in Fig. 1, are not very suitable for $\mathrm{AoC}$ implementation, particularly for lower $\mathrm{GHz}$ frequencies. Around six to nine metal layers are available in the $\mathrm{SiO}_{2}$ with interconnected-vias in a typical CMOS stack-up. The high relative permittivity of $\mathrm{Si}\left(\varepsilon_{\mathrm{r}}=11.9\right)$ attracts most of the antenna fields towards the substrate instead of being radiated in the air $[8,9]$. Further, the low resistivity $(\sim 10 \Omega-\mathrm{cm})$ of $\mathrm{Si}$ causes the loss of power in the Si substrate as heat. The poor radiation performance of the $\mathrm{AoC}$ is due to both, the high permittivity and conductivity. Besides, the $\mathrm{Si}$ substrate thickness $(300-700 \mu \mathrm{m})$ is also electrically large (particularly for mm-wave frequencies). Resultantly, surface wave modes get excited, which leads to the distortion of the radiation pattern $[10,11]$.

In order to improve the AoC radiation performance, two approaches have been reported extensively in the literature [8, 12-30]. First, it involves incorporating off-chip microwave lenses or superstrates. For instance, in [8], the gain of a $77 \mathrm{GHz}$ on-chip dipole antenna has been boosted by $10 \mathrm{~dB}$ through the hemispherical lens. In [18], a high-contrast superstrate (PREFERM FLX 1100) has been placed above the AoC, which leads to a boresight gain improvement by $4.5 \mathrm{~dB}$. In the second approach, either the lossy Si substrate is removed underneath the AoC or its properties are modified. For example, in [21], the $\mathrm{Si}$ beneath the AoC has been etched through micromachining and a gain of $4 \mathrm{dBi}$ is achieved at $85 \mathrm{GHz}$. Similarly, in [28], the $\mathrm{Si}$ resistivity has been selectively enhanced by Helium-3 ion irradiation, resulting in an improvement in radiation efficiency by $43 \%$. However, these methods are incompatible with CMOS processes and require complex post-fabrication steps, which not only increases the overall cost, but also adds alignment uncertainty and mechanical stability issues.

Alternatively, the Artificial Magnetic Conductors (AMC) can be employed to isolate the substrate and provide constructive reflection for the gain enhancement of AoC. Conventional implementation of AMC is to place the ground plane underneath the silicon substrate because of the thickness limitation of the $\mathrm{SiO}_{2}$, as shown in Fig. 2(a). Such a design was illustrated in [18], where the gain of the $71 \mathrm{GHz}$ on-chip monopole was boosted by $4.1 \mathrm{~dB}$. Nevertheless, in this situation, the AoC cannot be shielded completely from the 


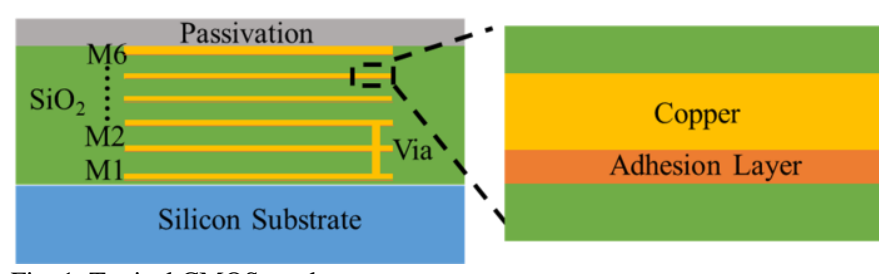

Fig. 1. Typical CMOS stack-up.

silicon substrate, and the undesired crosstalk between circuits and antennas still exists [11]. A better solution is to fully isolate the lossy Si substrate by realizing the AMC ground plane above the $\mathrm{Si}$, as shown in Fig. 2(b). However, this is dependent on the available $\mathrm{SiO}_{2}$ thickness, which is typically limited to $10-15 \mu \mathrm{m}$ and thus not suitable for the AMC realization as mentioned above. M. Nafe demonstrated an on-chip AMC with the ground plane above the silicon substrate, and the improvement of 8.4 $\mathrm{dB}$ in gain was achieved, however this was done at the expense of a thicker $\mathrm{SiO}_{2}$ layer of $40 \mu \mathrm{m}$ [31]. Nonetheless, contrary to other gain enhancement techniques, on-chip AMC can keep the system compact and provide an inexpensive solution due to its compatibility with the CMOS processes. The only bottleneck is that it is difficult to fit the AMC in the conventional thin $\mathrm{SiO}_{2}$ layer.

Another issue that affects the efficiency of AoC is the presence of thin adhesion layers (10 $\mathrm{nm}$ titanium or chromium) that are employed to provide good adhesion to the main metal layers (copper or gold). The surface resistance of these adhesion layers is large because of the skin effect, and thus AoC suffers from undesired ohmic losses [32].

These two issues related to AoC, i.e., fitting the AMC in conventional $\mathrm{SiO}_{2}$ thickness to isolate lossy $\mathrm{Si}$ substrate completely, and avoiding the ohmic loss due to the adhesion layers, are tackled in this paper. An attempt to introduce Metallic Posts (MP) by employing the vias in the stack-up, thereby reducing the AMC thickness, has been carried out in [33], while the sufficient insight into its working principle and comprehensive parametric analysis is presented in this paper. The MP behave as slow-wave structures, which affect the phase velocity and consequently helps in AMC thickness reduction. Furthermore, another novel thickness reduction structure (TRS), working the available metal layers into Embedded Guiding Structures (EGS), has been proposed in this paper. The EGS modifies the path of electric fields inside the AMC, making the AMC look electrically thicker. In contrast, the EGS provides more thickness miniaturization as compared to the MP. Finally, a $16 \mu \mathrm{m}$ ultra-thin AMC is realized with a gain improvement of $9.15 \mathrm{~dB}$ as compared to a standalone antenna. In the fabrication, adhesion layers have been completely omitted so ohmic losses due to them have been avoided without losing the required adhesion for the main metal layers. As per author's best knowledge, this is the first demonstration of EGS for AMC realization in an on-chip environment to boost AoC gain and efficiency. The measured $\mathrm{AoC}$ gain is one of the highest in the literature that has been achieved without off-chip components or post-fabrication processes.

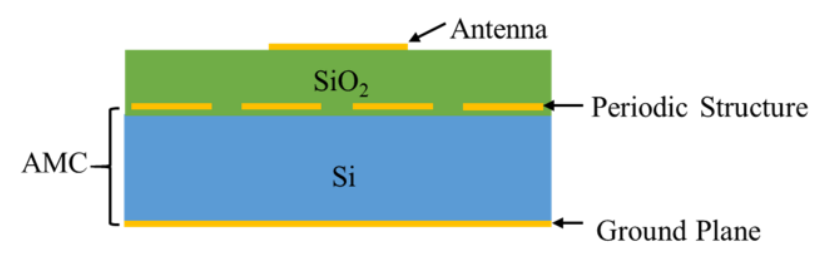

(a)

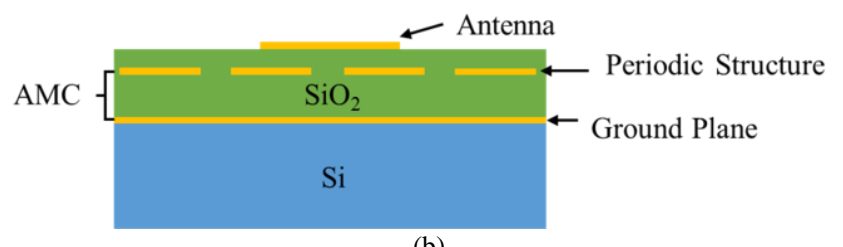

(b)

Fig. 2. Implementation approaches of AMC: the ground plane is (a) below Si substrate, and (b) above Si substrate.

\section{ON-Chip Monopole ANTENNA WITH REFERENCE AMC}

On-chip antennas are mostly designed to be horizontally placed on the top metal layer of the stack-up. This is because the top metal layer allows larger metal thickness which is beneficial for antenna's performance and also this arrangement enables the antenna to radiate directly into the air. According to the image theory, radiation performance of a horizontally placed antenna could be enhanced by a Perfect Magnetic Conductor (PMC) surface that can produce an image current in the same direction [34]. Since PMC surfaces do not exist in nature, AMC surfaces are developed to mimic the effect of PMC for a certain frequency range. Conventional AMC comprises three layers: a periodic metallic layer, a dielectric slab and a ground plane. The square patch based AMC is one of the most commonly used structures which demonstrates a low return loss and wide operating bandwidth [35]. So, in this article, a square patch based AMC is selected for further investigation. Throughout this work, the FEM-based 3-D full-wave electromagnetic (EM) solver, ANSYS High Frequency Structure Simulator (HFSS), has been used for simulations.

To isolate the silicon substrate completely from the antenna, the ground plane of the AMC is located on the top of the silicon substrate. The $\mathrm{SiO}_{2}$ layer has a dielectric constant of 4.0 and houses the periodic square patch metallic layer. The metal layers are modelled by copper with a conductivity of $5.8 \times 10^{7}$ $\mathrm{S} / \mathrm{m}$. Besides, considering a chip with acceptable dimensions, the reference AMC model is simulated as a $4 \times 4$ finite surface, as shown in Fig. 3. Except the AMC thickness $t_{A M C}$, the other geometrical parameters are fixed for further investigation. According to the simulated result, shown in Fig. 3(b), the reflection phase of the AMC crosses zero degrees at $94 \mathrm{GHz}$ when the AMC thickness becomes $27 \mu \mathrm{m}$.

In order to study the reflection and isolation performance of the reference AMC, a planar monopole antenna is placed on top of the AMC, as shown in Fig. 4. To test it with a probe, the antenna is fed by a coplanar waveguide (CPW); while in a practical system, the antenna can get signals fed through the vias in the standard CMOS stack-up. The AoC dimensions are given in Table I. According to the simulated results shown in 


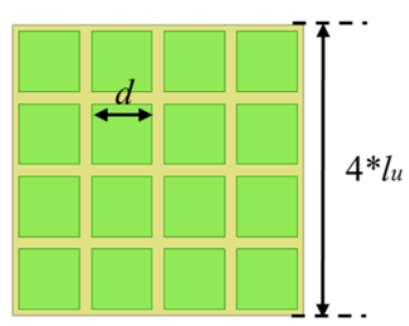

(a)

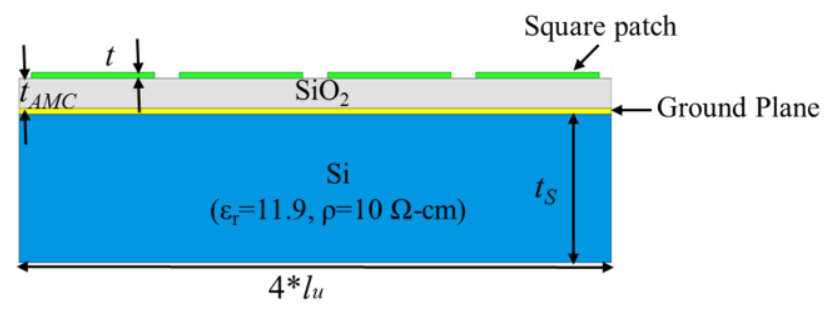

(c)

Fig. 3. $4 \times 4$ finite patch-based reference AMC: (a) Top view, $d=590, l_{u}=600$, (b) reflection phase, and (c) side view, $t=0.5, t_{A M C}=27, t_{S}=500$ (all in $\mu \mathrm{m}$ ).

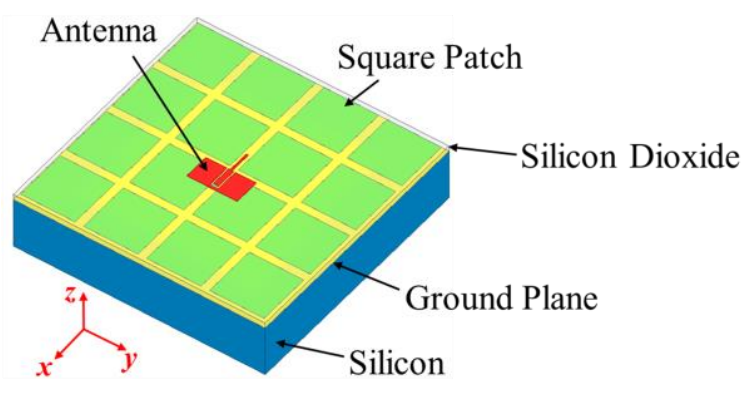

(a)

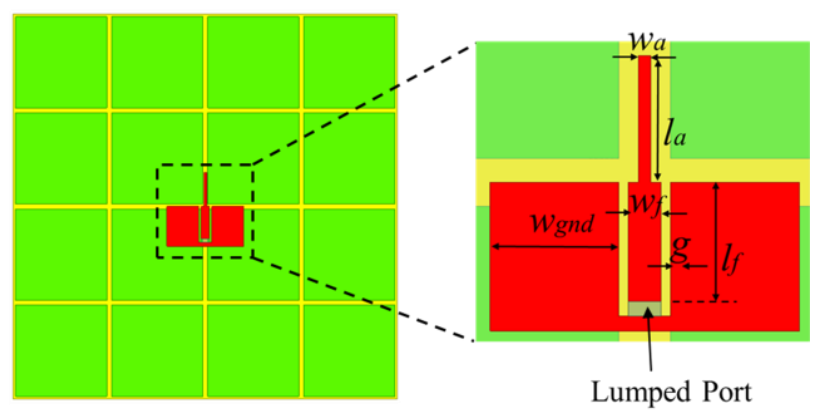

(b)

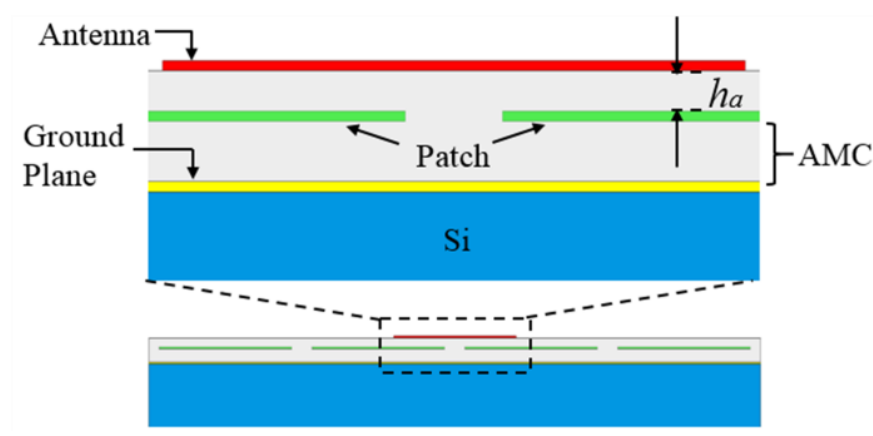

(c)

Fig. 4. Reference AMC-backed monopole antenna: (a) HFSS model, (b) top view, and (c) side view.

Fig. 5, it is well-matched at $94 \mathrm{GHz}$ with a return loss of 18.8 dB. It demonstrates boresight radiation with a gain of $4.5 \mathrm{dBi}$
TABLE I

DIMENSIONS OF THE ANTENNA BACKED BY DIFFERENT AMC (ALL IN $\mu \mathrm{m})$

\begin{tabular}{|c|c|c|c|c|c|c|c|}
\hline & $l_{a}$ & $l_{f}$ & $w_{a}$ & $w_{f}$ & $w_{\text {gnd }}$ & $h_{a}$ & $g$ \\
\hline Ref. AMC & 213 & 200 & 20 & 50 & 200 & 2.5 & 15 \\
\hline $\begin{array}{c}\text { AMC + MP } \\
\text { [33] }\end{array}$ & 200 & 181 & 20 & 50 & 200 & 1.5 & 15 \\
\hline AMC + EGS & 380 & 100 & 10 & 15 & 160 & 1.5 & 25 \\
\hline
\end{tabular}

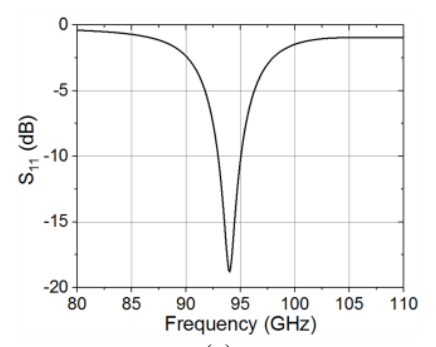

(a)

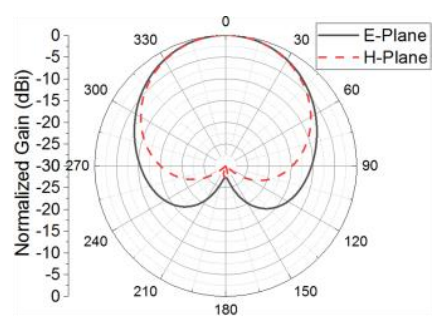

(b)
Fig. 5. Simulated results of the monopole antenna backed by reference AMC: (a) $\mathrm{S}_{11}$, (b) Normalized radiation pattern in E-plane ( $x z$-plane) and H-plane (yz-plane).

and radiation efficiency of $45 \%$ at $94 \mathrm{GHz}$ due to the in-phase reflection provided by the reference AMC.

\section{AMC THICKNESS REDUCTION}

For the AMC to work properly at $94 \mathrm{GHz}, \mathrm{a} \mathrm{SiO}_{2}$ layer thickness of $27 \mu \mathrm{m}$ is required, as has been determined through EM simulations in the previous section. This thickness requirement is too high for the stack-up of current CMOS processes. Therefore, thickness miniaturization techniques need to be introduced in the AMC design to reach an acceptable oxide thickness. In this section, two kinds of thickness reduction structures (TRS) are introduced and investigated for a single AMC unit cell as well as the $4 \times 4$ finite AMC-backed AoC.

\section{A. Metallic Posts (MP)}

For a conventional patch based AMC, the equivalent model comprises a capacitance $C_{o}$ with a shunt inductance $L_{o}$, where $C_{o}$ is the capacitance between adjacent patches, while $L_{o}$ refers to the inductor due to the backside ground plane [36]. Inspired by slow-wave structures, the vias available in the oxide layer can be utilized to form MP, as shown in Fig. 6(a), thus additional series inductors $L_{M P}$ and shunt capacitors $C_{M P}$ can be introduced, where $L_{M P}$ is related to its height, and $C_{M P}$ is the capacitance between the patch and the MP top surface. The phase velocity $v_{p}$ of the waves propagating between the patch and the ground plane is inversely correlated with the additional reactance as given in (1) [37]

$$
v_{p} \propto \frac{1}{\sqrt{2 L_{M P} \times 2 C_{M P}}}
$$

Through this additional reactance, the phase velocity is reduced, and equivalently the electrical thickness of the AMC is increased. Considering the ohmic losses proportional to the metal volume in each unit cell, the loss resistance would be quite large if too many vias are introduced. Therefore, in the unit cell design, two vias originate from the ground plane but they are not connected with the patch, forming MP. It is noted that the monopole antenna in Fig. 4 has the radiating arm in the 


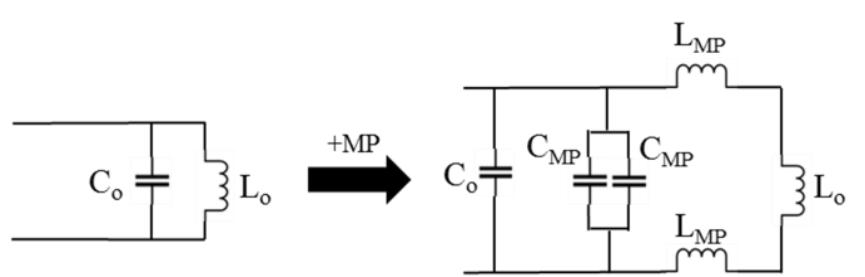

(a)

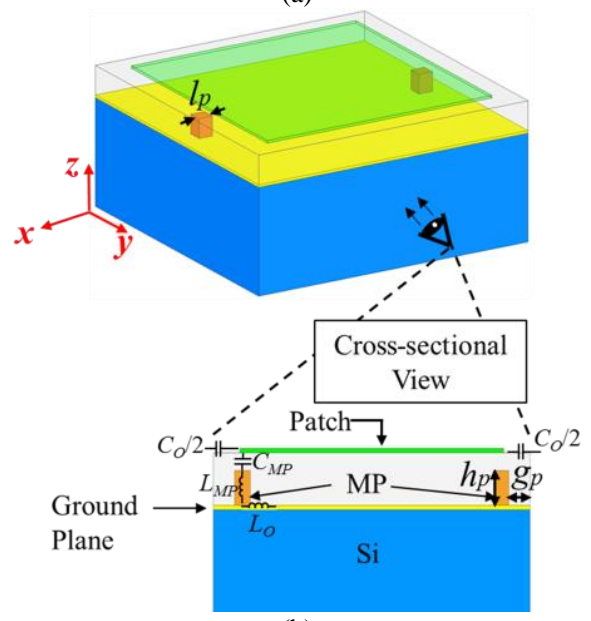

(b)

Fig. 6. Patch-based AMC with MP (a) equivalent circuit, (b) two MPs are introduced in one AMC unit cell.

$x$-direction, resulting in the electric fields excited in the AMC mainly vary along the $x$-direction. Therefore, the two MP are placed along the $x$-direction symmetrically, with a distance of $g_{p}$ from the near side unit edge, as shown in Fig. 6(b).

A comprehensive study investigating the effect of the geometrical parameters of the metallic posts on the AMC resonance frequency $f_{A M C}$ is conducted to achieve the best results. Typically, the via size $l_{p}$ is often a fixed value but the degrees of freedom for design are its height, $h_{p}$, and location, $g_{p}$. To investigate the effect of these design parameters of MP on the AMC resonance frequency, $l_{p}$ and $t_{A M C}$ are fixed as 6.7 and $13 \mu \mathrm{m}$ respectively. Firstly, in Fig. 7(a), $g_{p}$ varies from 20 to $110 \mu \mathrm{m}$, while $h_{p}$ is defined as $10.5 \mu \mathrm{m}$. It is found that $f_{A M C}$ decreases gradually until $g_{p}$ reaches $70 \mu \mathrm{m}$ with a sharp depression at $g_{p}=73 \mu \mathrm{m}$, after which it starts to increase. As Fig. 8(a) and (b) show, the current on the patch is mainly distributed along the upper and lower side edge, which is opposite to the situation of the current in the ground plane. This can be explained as follows. When $g_{p}$ increases, the additional inductance increases significantly, while the capacitance between the patch and the top surface of the posts decreases a little. Thus, the rising inductance dominates in (1), which leads to a decreasing $f_{A M C}$. However, when $g_{p}$ becomes larger than 73 $\mu \mathrm{m}$, the inductance increases slightly, but the capacitance decreases significantly, which means the decreasing capacitance is dominant in (1) and, thus, the resonance frequency rebounds.

Also, MP height, $h_{p}$, is varied from 1 to $10.5 \mu \mathrm{m}$ while keeping the $g_{p}$ as $73 \mu \mathrm{m}$. The result shown in Fig. 7(b) illustrates that $f_{A M C}$ demonstrates a constant downward trend when $h_{p}$ is increased. This is because when $h_{p}$ increases, the current path is extended and the MP top surface becomes closer

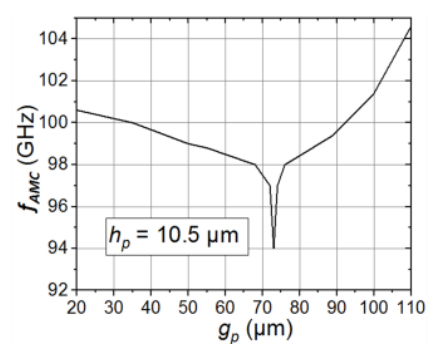

(a)

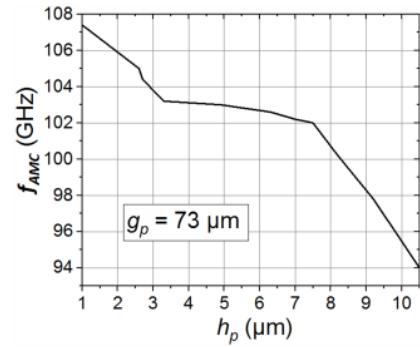

(b)
Fig. 7. Tuning effect of MP on the AMC resonance frequency: Sweep of (a) $g_{p}$, and (b) $h_{p}$.
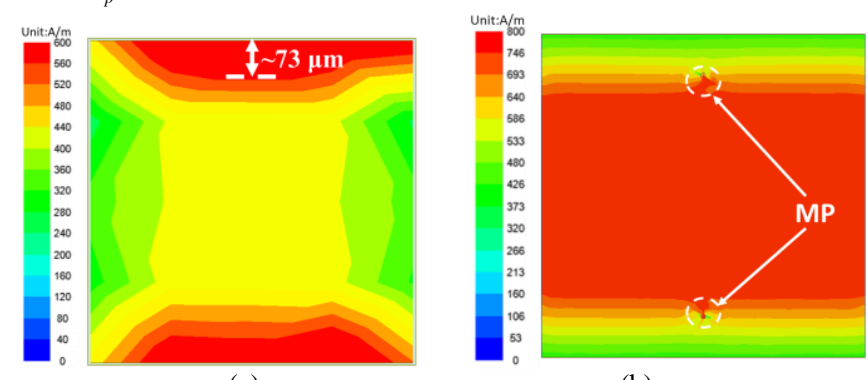

(a)

(b)

Fig. 8. Current distribution on (a) the square patch, and (b) the ground plane.

to the patch, thus the introduced inductance and capacitance both increase. According to (1), the phase velocity and the resonance frequency are both reduced.

From the results of the parametric sweep, it is clear that the $\mathrm{MP}$ can tune the AMC resonance frequency. By implanting two MP in each unit cell of the $4 \times 4$ finite patch-based AMC, as illustrated in Fig. 9, the AMC can resonate at $94 \mathrm{GHz}$ with a thickness of $18 \mu \mathrm{m}$. Compared to the reference AMC, $t_{A M C}$ is reduced by 33\%. As reported in [33], the $94 \mathrm{GHz}$ monopole antenna backed by this thin AMC with the dimensions given in Table I radiates in the boresight direction with a gain of $1.2 \mathrm{dBi}$ and radiation efficiency of $23 \%$.

\section{B. Embedded Guiding Structures (EGS)}

The stack-up of a standard CMOS process provides about six to nine metal layers inside the oxide. These metal layers are embedded in the dielectric part of the AMC, and can be smartly used to reduce its resonance frequency. As Fig. 11(a) shows, the electric fields around the EGS have to make a detour so that the electrical thickness of the oxide layer appears to be larger. Considering the influence of the resistance, only one metal layer is employed in the AMC unit cell study, as shown in Fig. 11(b). Similar to the MP, there are two symmetric guiding structures implemented into one AMC unit cell with planar dimensions of $l_{g}$ and $w_{g}$. The gap between the structures and the edge of the unit cell is defined as $g_{g}$ while $h_{g}$ refers to the distance of the EGS to the ground plane. The equivalent circuit model of a conventional patch-based AMC is a parallel LC circuit [36]. Because of the EGS, new distributed capacitors and an inductor, as shown in Fig. 11(b), are introduced. The capacitance between the EGS and the patch is defined as $C_{E G S}$; the capacitance between the EGS and the ground plane is $C_{E G S G}$; the capacitance between the EGS of adjacent unit cells is modelled as $C_{E G S O}$. All introduced capacitances are in parallel with $C_{o}$ in the equivalent circuit model [38]. The inductor on the EGS, $L_{E G S}$, appears on the branch of $C_{E G S O}$. The complete 


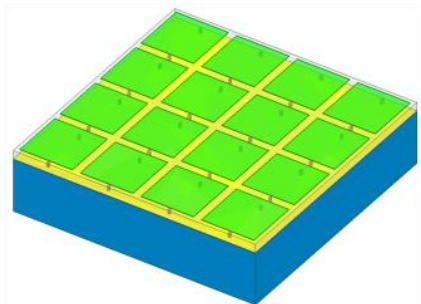

(a)

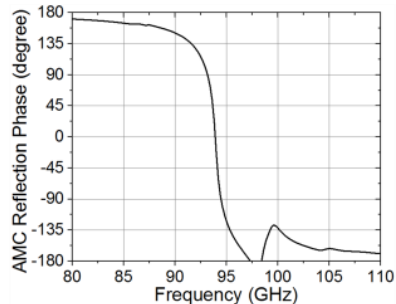

(b)

Fig. 9. $4 \times 4$ finite patch-based AMC with MP: (a) proposed model, and (b) phase of the reflection coefficient.

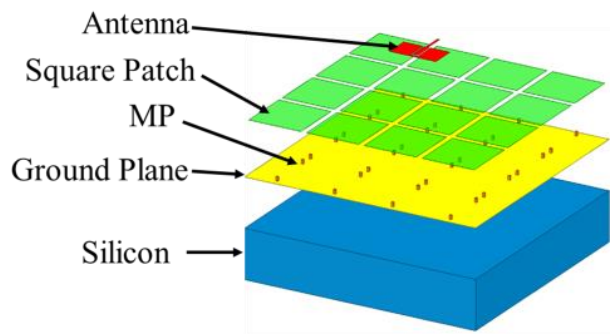

(a)

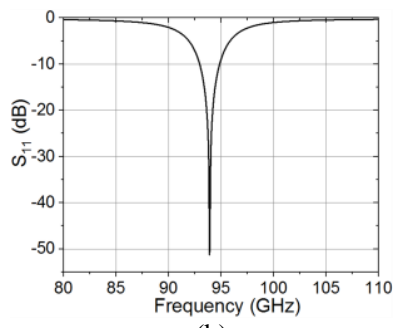

(b)

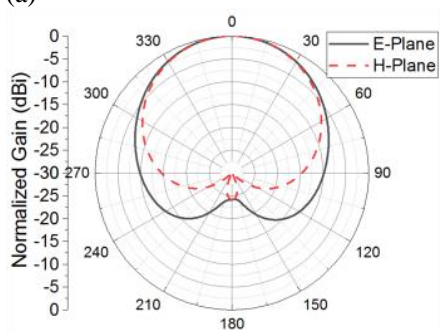

(c)

Fig. 10. AMC with MP backed monopole antenna: (a) 3D exploded view, and simulated (b) $\mathrm{S}_{11}$, (c) normalized radiation pattern [33].

equivalent circuit of the AMC with EGS is shown in Fig. 11(c). These additional capacitors and inductors provide further flexibility to tune the AMC resonance frequency.

To ascertain the effect of guiding structure parameters on the AMC's thickness reduction, a parametric sweep of each geometrical parameter of the embedded structures is considered. The AMC thickness $t_{A M C}$ is $13 \mu \mathrm{m}$. Considering fabrication limitations of the in-house CMOS-compatible process, the gap between the structures and the unit edge $g_{g}$ is varied from 5 to $45 \mu \mathrm{m}$, the height of the structures $h_{g}$ is ranged from 1 to $10.5 \mu \mathrm{m}$, the length of the structures $l_{g}$ is tuned from 200 to $390 \mu \mathrm{m}$ and the width $w_{g}$ is varied from 50 to $250 \mu \mathrm{m}$.

According to Fig. 12(a), the gap $g_{g}$ is varied while the height, length and width of the EGS are fixed. It is noted that the resonance frequency is proportional to $g_{g}$. This is because the electric fields in the $\mathrm{SiO}_{2}$ concentrate around the side edges, as Fig. 13 shows, thus the bending of the electric field path decreases with $g_{g}$ increasing.

As Fig. 12(b) shows, when $g_{g}, l_{g}$ and $w_{g}$ are fixed, $f_{A M C}$ goes down till the height $h_{g}$ reaches $4 \mu \mathrm{m}$, and then it rebounds. According to the electric field distribution in the $\mathrm{SiO}_{2}$, the fields are stronger near the patch. When $h_{g}$ increases, more electric fields detour due to the EGS, thus $f_{A M C}$ decreases. However, when EGS is too close to the patch, the embedded structures provide reflection instead of guidance, which is similar to what the ground plane does. For instance, the vectors of the electric fields near the side edge of the EGS, when $h_{g}=8 \mu \mathrm{m}$, are shown

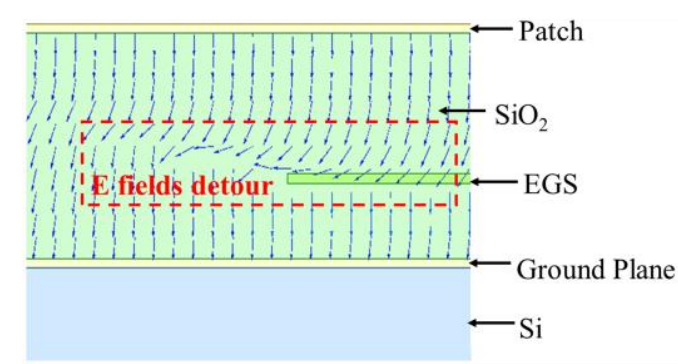

(a)

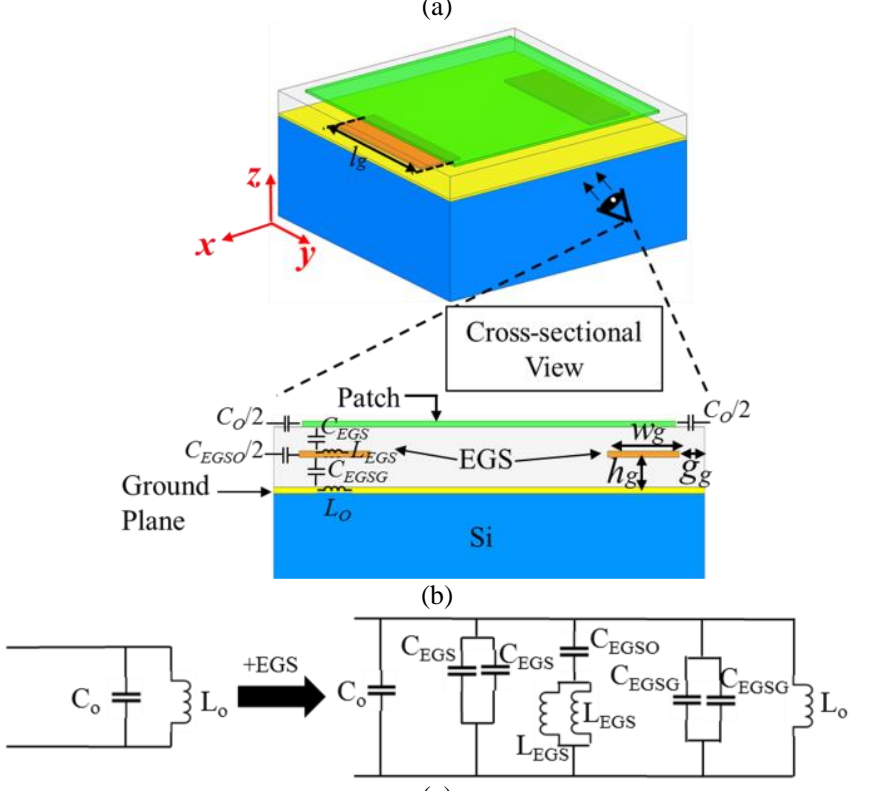

(c)

Fig. 11. Patch-based AMC unit cell with EGS: (a) Electric fields detour around EGS, (b) two EGSs are introduced in one AMC unit cell, (c) equivalent circuit model.

in Fig. 14(a). It can be noticed that when $h_{g}$ reaches $8 \mu \mathrm{m}$, the electric fields near the embedded structures do not detour. Contrarily, the reflection characteristic of the EGS causes the decrease in the electrical thickness, resulting in the increasing trend of the resonance frequency when $h_{g}$ is larger than $4 \mu \mathrm{m}$.

In Fig. 12(c) and (d), $f_{A M C}$ shows a sharp decrease and then a rise with the increasing dimensions, $l_{g}$ and $w_{g}$. When the length and width of the structure is less than 220 and $90 \mu \mathrm{m}$ respectively, the propagation of more proportion of the electric fields meanders. However, when the dimensions of the embedded structures become too large, e.g. $l_{g}=300 \mu \mathrm{m}$ or $w_{g}=$ $180 \mu \mathrm{m}$, the detour of the electric fields near the EGS disappears, as Fig. 14(b) and (c) illustrate, which causes the rebound of $f_{A M C}$.

The EGS is introduced in the $4 \times 4$ finite patch-based AMC, as shown in Fig. 15(a), where the structures on the chip edges have a gap of $g_{g}$ away from the edge and dimensions of $l_{g}$ and $w_{g}$, while the EGS between each adjacent unit cell along $x$ direction is continuous with a length of $l_{g}$ and width of $2 \times\left(g_{g}+\right.$ $w_{g}$ ). According to Fig. 15(b), when $g_{g}=5 \mu \mathrm{m}, h_{g}=9.5 \mu \mathrm{m}, l_{g}=$ $310 \mu \mathrm{m}, w_{g}=100 \mu \mathrm{m}$, the finite AMC with EGS has zero-phase of reflection at $94 \mathrm{GHz}$, while the oxide thickness of the AMC with proposed EGS is only $16 \mu \mathrm{m}$, which means a $41 \%$ reduction in thickness as compared to the reference AMC (without EGS). 


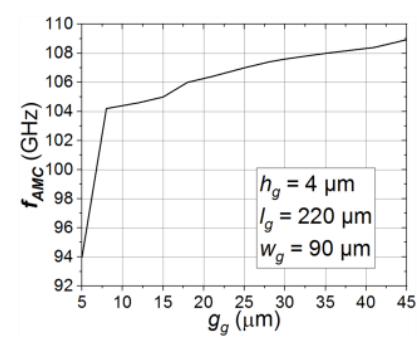

(a)

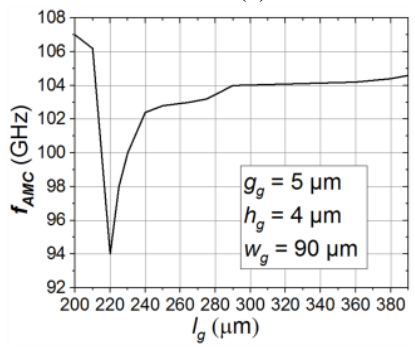

(c)

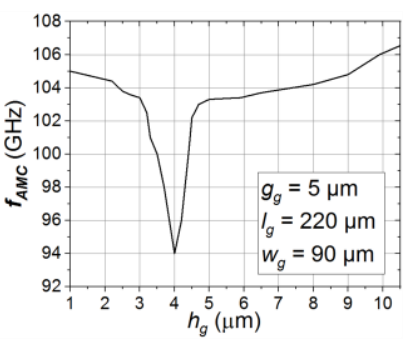

(b)

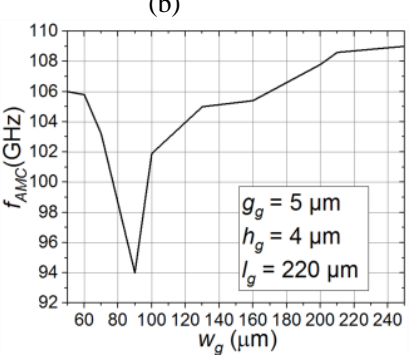

(d)

Fig. 12. Tuning effect of EGS on the AMC resonance frequency: Sweep of (a) $g_{g}$, (b) $h_{g}$, (c) $l_{g}$, and (d) $w_{g}$

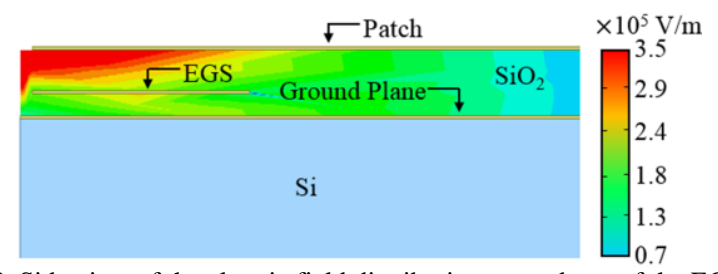

Fig. 13. Side view of the electric field distribution around one of the EGS.

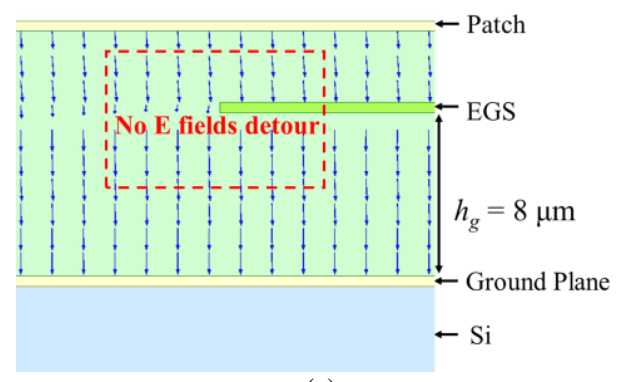

(a)

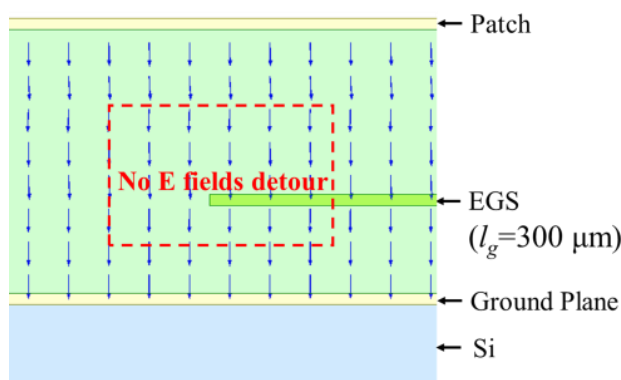

(b)

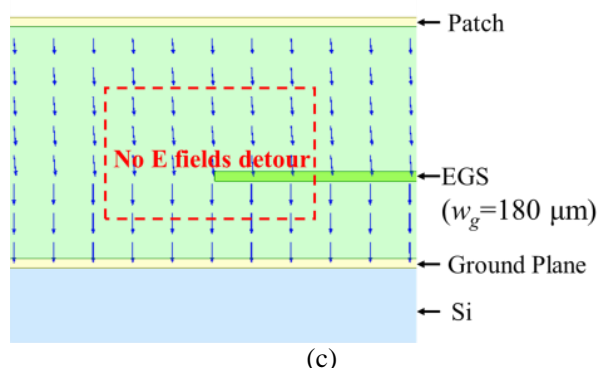

(c)

Fig. 14. Vectors of the electric field in the silicon dioxide: (a) $h_{g}=8 \mu \mathrm{m}$ (b) $l_{g}=$ $300 \mu \mathrm{m}$, and (c) $w_{g}=180 \mu \mathrm{m}$.

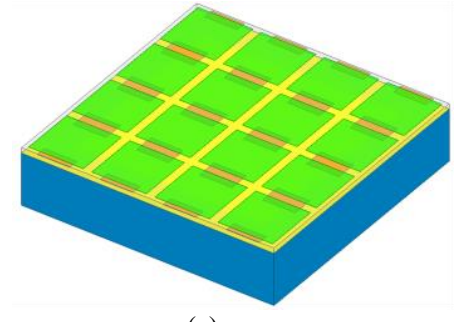

(a)

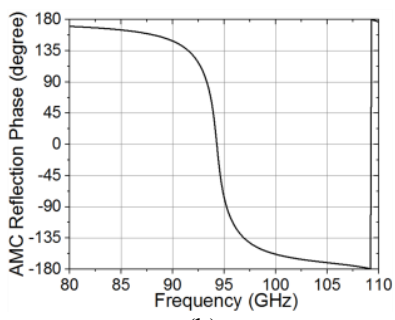

(b)
Fig. 15. $4 \times 4$ finite patch-based AMC with EGS: (a) proposed model, and (b) phase of the reflection coefficient.

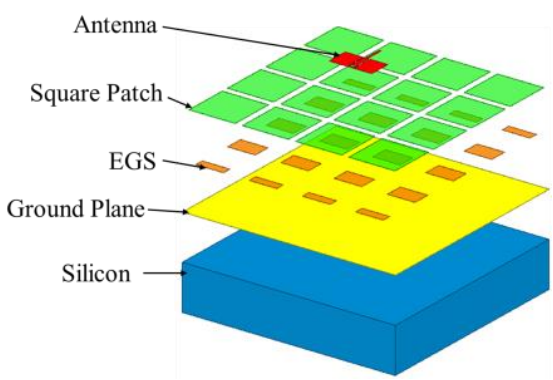

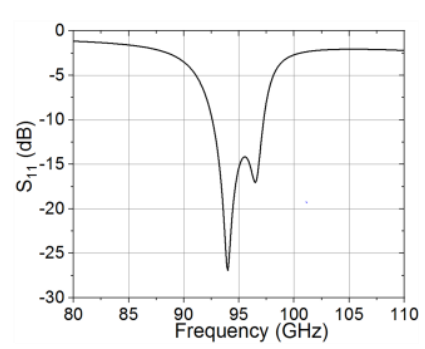

(b)

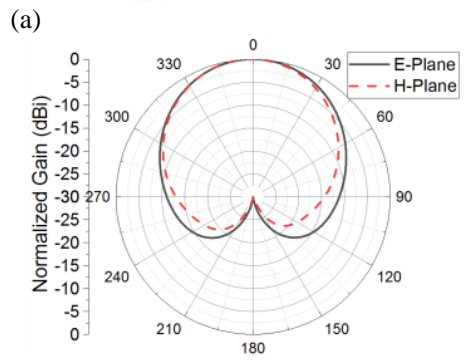

(c)
Fig. 16. AMC with EGS backed monopole antenna: (a) 3D exploded view, and simulated (b) $S_{11}$, (c) normalized radiation pattern.

Like the previous cases, a monopole antenna has been realized above the proposed AMC with EGS, as shown in Fig. 16 (a). The AoC has a good impedance matching at $94 \mathrm{GHz}$ with a return loss better than $25 \mathrm{~dB}$, while it provides a boresight gain of $5.08 \mathrm{dBi}$ with a radiation efficiency of $50 \%$ (Fig. 16 (b) and (c)).

It is noted that another resonance appears at $96.5 \mathrm{GHz}$. This is caused by the EGS on the left and right edges, shown in circles in Fig. 17(a). This can be clearly seen in the current distribution on the EGS layer at $96.5 \mathrm{GHz}$. To further verify the origin of the second resonance, the lengths and widths of the circled EGS, marked as $l_{c}$ and $w_{c}$, have been varied by 20 and 4 $\mu \mathrm{m}$ respectively, while the other EGS dimensions are not changed. The results of these simulations, shown in Fig. 17(b) and (c), confirm that the second resonance shifts by 1 and 0.8 $\mathrm{GHz}$ respectively, but there is no change in the main resonance at $94 \mathrm{GHz}$. The radiation pattern, shown in Fig. 16 (c), is for the $94 \mathrm{GHz}$ band as that is the resonant frequency of the radiator.

\section{Comparison of Thickness Reduction Techniques}

Conventionally, the vias are used to connect multiple metal layers in the CMOS stack-up. However, in this work, the vias and metal layers have been utilized to reduce the thickness of the AMC. Two finite AMC surfaces implemented with different TRS have been proposed in the previous sections. In a standard CMOS process, the designer must carefully follow the foundry rules, typically called design rule check (DRC). For 

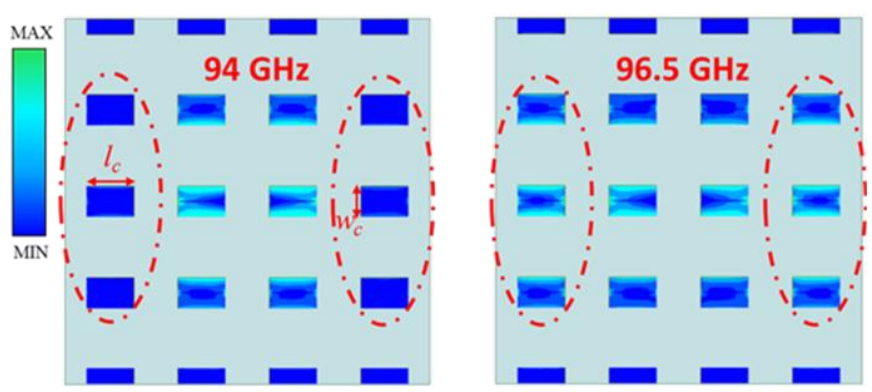

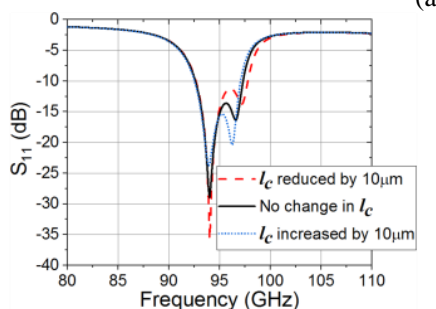

(b) (a)

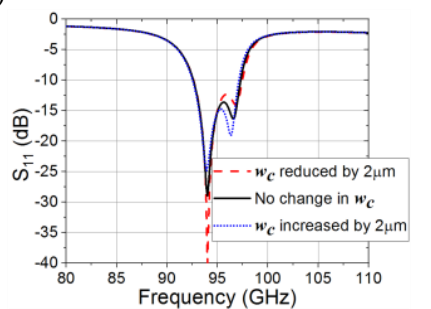

(c)
Fig. 17. Resonance at $96.5 \mathrm{GHz}$ in the case of AMC with EGS backed monopole antenna: (a) current distribution on EGS layer (b) resonance shifts with different lengths and, (c) widths of the circled EGS.

example, rules such as metal width, spacing between two conductors, etc. must be followed strictly in order to qualify for fabricating the design in a particular foundry. If we compare two TRS, it is obvious that MP approach is simpler in terms of design, DRC and fabrication aspects.

An identical monopole antenna is placed $2.5 \mu \mathrm{m}$ above these AMC structures with MP and EGS respectively. Table II summarizes the AMC thicknesses and radiation performance.

It is clear that the implementation of EGS within the AMC leads to a greater reduction in thickness. The monopole antenna integrated with the EGS based AMC shows higher gain and radiation efficiency $(5.08 \mathrm{dBi}$ and $50 \%)$. The antenna backed by the MP based AMC has relatively low gain, due to the undesired high conductor loss. Using HFSS field calculator, power loss analysis of MP based AMC is performed for a fixed input power of $1 \mathrm{~W}$, and conductor loss for each element is listed in Table III. It is evident that when AMC is integrated with MP, more proportion of energy is thermally lost in antenna, thus the radiation performance of this design is not that good.

Compared to the reference AMC, the AMC with EGS has the least thickness and offers the best gain. Therefore, it is selected for further investigation of the optimum AMC size. As Fig 18(a) shows, the peak gain of the on-chip monopole antenna positively correlates with the number of AMC unit cells. When there is no AMC, the gain of the standalone AoC is about -3.3 $\mathrm{dBi}$ and the radiation patterns are distorted (Fig 18(b)) Considering that, typically, the radiation pattern of a CPW-fed planar monopole antenna is symmetric in air, the lossy silicon substrate must be responsible for the slight distortion in the radiation pattern of the standalone antenna [39]. By implementing the AMC to isolate the antenna from the silicon substrate, the gain of AoC improves as the number of AMC unit cells increases, till it saturates when the number of unit cells reaches 16. Hence, the AMC size of $4 \times 4$ is selected as a
TABLE II

COMPARISON OF THREE KINDS OF FINITE ON-CHIP AMC SURFACES

\begin{tabular}{|c|c|c|c|c|}
\hline & $\begin{array}{c}t_{A M C} \\
(\mu \mathrm{m})\end{array}$ & $\begin{array}{c}\text { Thickness } \\
\text { Reduction }\end{array}$ & $\begin{array}{c}\text { Gain of AoC } \\
(\mathrm{dBi})\end{array}$ & $\begin{array}{c}\text { Radiation } \\
\text { Efficiency }\end{array}$ \\
\hline Ref. AMC & 27 & & 4.5 & $45 \%$ \\
\hline AMC + MP & 18 & $33 \%$ & 1.2 & $23 \%$ \\
\hline AMC + EGS & 16 & $41 \%$ & 5.08 & $50 \%$ \\
\hline
\end{tabular}

TABLE III

CONDUCTOR LOSS PROPORTION OF EACH PART OF AMC-BACKED AOC

\begin{tabular}{|c|c|c|c|c|}
\hline & Antenna & Patch & TRS & Ground Plane \\
\hline Ref. AMC & $17.9 \%$ & $27.3 \%$ & & $9.8 \%$ \\
\hline AMC + MP & $27.6 \%$ & $36.7 \%$ & \multicolumn{2}{|c|}{$12.7 \%$} \\
\hline AMC + EGS & $5.1 \%$ & $22 \%$ & $4.7 \%$ & $18.2 \%$ \\
\hline
\end{tabular}

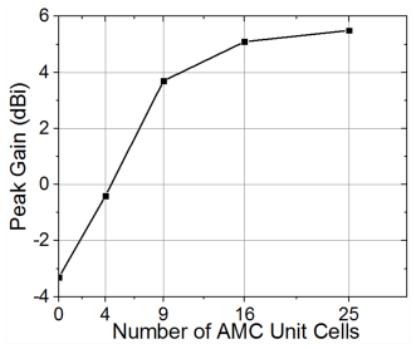

(a)

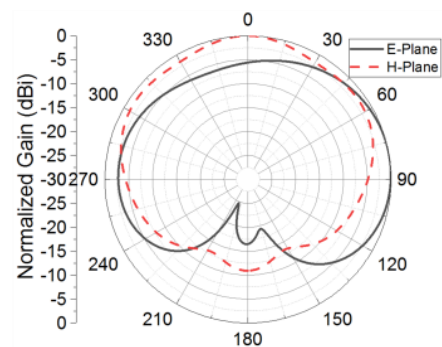

(b)
Fig. 18. Investigation on the AMC with EGS surface: (a) Peak gain of the AOC versus the number of AMC unit cells, and (b) distorted radiation pattern of a standalone antenna.

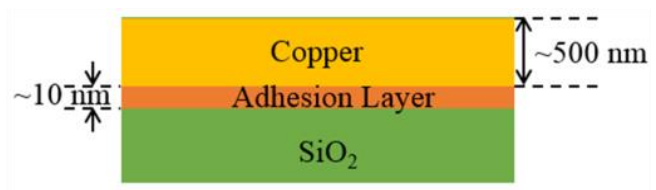

(a)

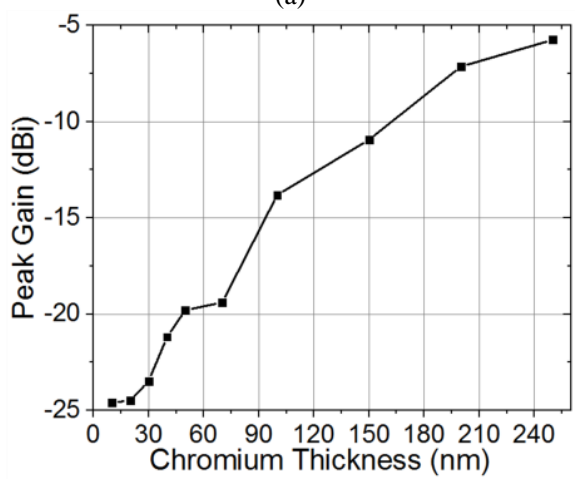

(b)

Fig. 19. (a) Adhesion layers bind the oxide with copper. (b) Peak gain versus the chromium film thickness.

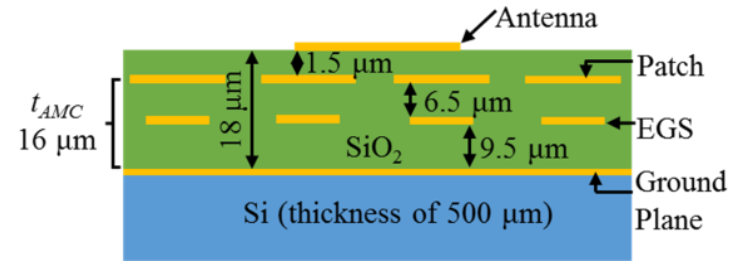

Fig. 20. Stack-up of the proposed structure.

compromise between the overall chip size and the gain of the antenna. 


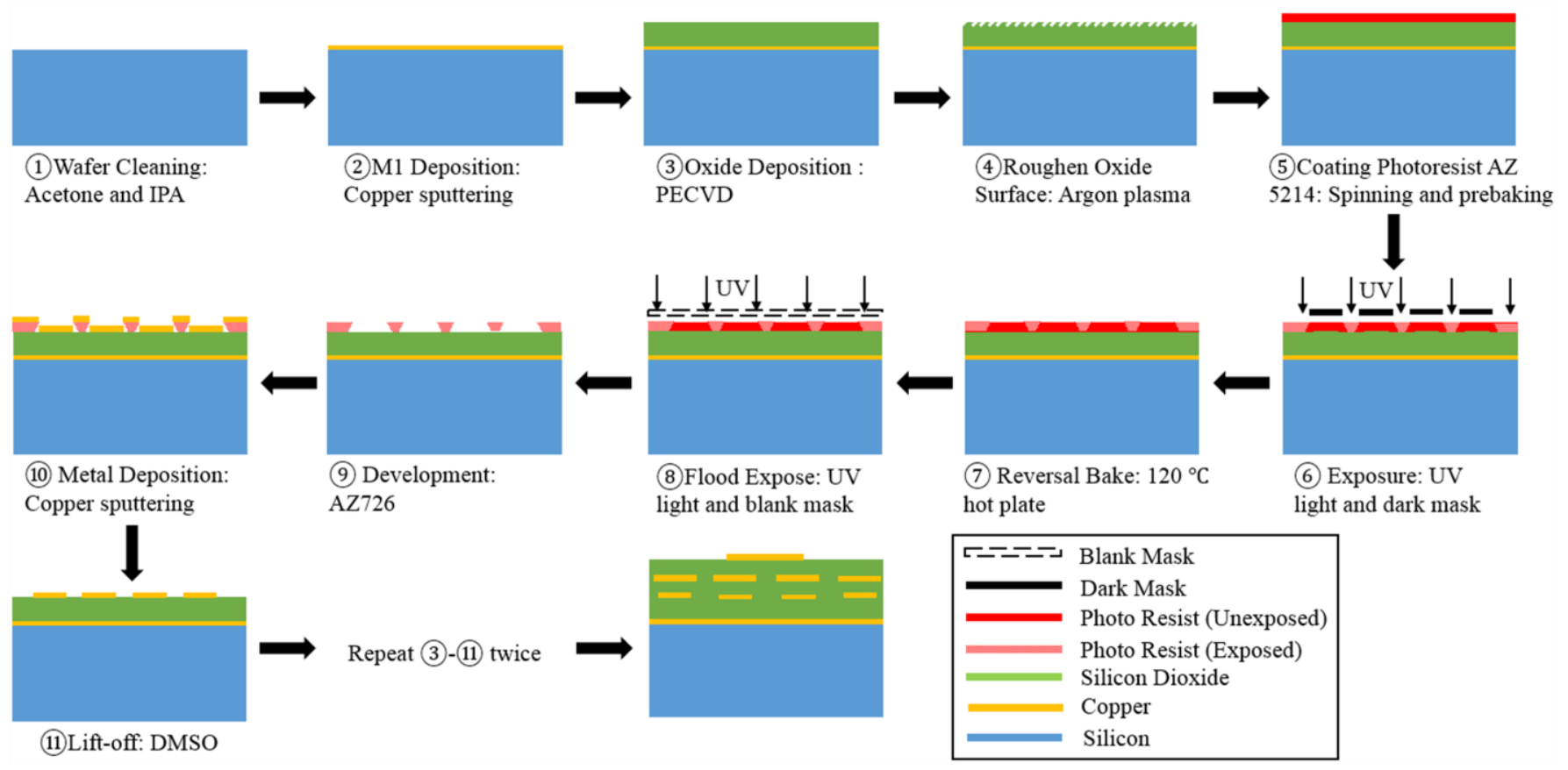

Fig. 21. Fabrication process without adhesion layers of the BEOL part.

\section{FABRICATION AND MEASUREMENT}

\section{A. Fabrication without Adhesion Layers}

The on-chip monopole antenna backed by a $4 \times 4$ thin AMC with EGS is selected for fabrication as it exhibits the highest gain for the thinnest oxide layer. During the deposition of the patterned metal layer, specialized adhesion layers, composed of chromium or titanium, are always used in a typical fabrication process, as shown in Fig. 19. This is to improve the adhesion of the metals (copper or gold) to the oxide. The usage of the adhesion films helps the buildup of noble metal in device fabrication, but it reduces the average conductivity of metal layers and negatively affects the AMC and antenna radiation. It is noted that when extremely thin AMC thickness is required, the resistance corresponding to the conductor loss plays a significant role that could affect the zero-degree reflection phase property by causing PEC-like effect on the AMC [40]. It is observed that a $10 \mathrm{~nm}$ chromium film modelled at the lower surface of each copper layer as the adhesion layer, causes the antenna gain to reduce to $-24.6 \mathrm{dBi}$ and radiation efficiency to less than $0.1 \%$. To study the effect of the adhesion layer thickness on the AoC radiation, the copper layer is fixed as 500 $\mathrm{nm}$, while the thickness of the chromium adhesion film is varied from 10 to $250 \mathrm{~nm}$. According to the simulated results, shown in Fig. 19(b), the peak gain shows a direct proportionality to the thickness of the chromium film. This is because the surface resistance is inversely proportional to the chromium film thickness when it is much thinner than the skin depth of chromium $(595 \mathrm{~nm}$ at $94 \mathrm{GHz})$. Therefore, the conduction loss caused by the adhesion films decreases with the chromium thickness increasing. Nevertheless, the gain is still unideal even if the chromium thickness reaches $250 \mathrm{~nm}$. Therefore, to realize a fabricated prototype that exhibits the enhanced gain and radiation performance as proposed in

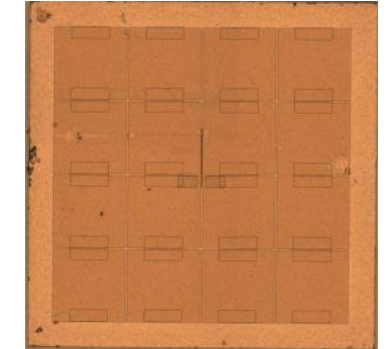

(a)

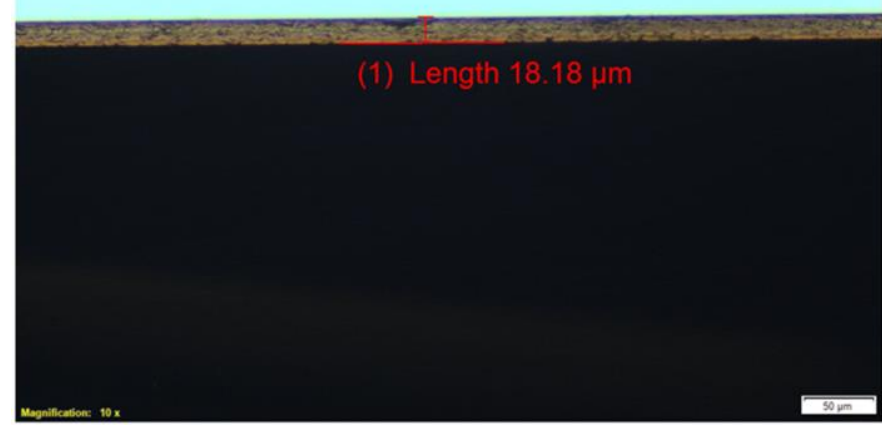

(c)

Fig. 22. Fabricated sample: (a) Top view from a microscope. (b) Dimensional measurement with a Vernier caliper. (c) Cross-sectional view from the microscope.

simulations, the adhesion layers cannot be involved, but certain modifications to the fabrication process are required to help the copper layers still bond adequately to the oxide.

The stack-up of the proposed structure of the monopole antenna backed by ultra-thin AMC with EGS is depicted in Fig. 20 , where the ground plane is supposed to fully cover the substrate, the EGS is designed at $9.5 \mu \mathrm{m}$ above the ground plane, while the periodic patch layer of the finite AMC has a gap of $6.5 \mu \mathrm{m}$ above the patch layer, and the planar monopole antenna is located $1.5 \mu \mathrm{m}$ above the patch layer.

The back end of line (BEOL) process is visually shown in Fig. 21. To maintain the copper layer on $\mathrm{SiO}_{2}$ without the 
adhesion layer deposition, two things need to be taken care of. On the one hand, it is essential to improve the adhesion of the copper to the oxide, while on the other hand, during the lift-off step of the lithography, the solvent must contact the residual photoresist easily, thereby preventing the patterned copper from exfoliation that would be caused by conventional intensive ultrasonic lift-off. It can be noticed that prior to the lithography, in the step 4, the bombardment of argon atoms can make the surface of $\mathrm{SiO}_{2}$ rough, thereby enhancing the friction force of the oxide to the copper and consequently improving the adhesion. After that, the wafer is coated with AZ 5214 image reversal photoresist whose ideal thickness is $1.6 \mu \mathrm{m}$, slightly larger than thrice of the thickness of the copper film. Then, the solidified photoresist is treated by dark-mask-covered exposure and the area except intended pattern is exposed. Next, the reversal baking with critical condition $\left(120^{\circ} \mathrm{C}\right.$ for two minutes) and flood exposure makes the unexposed intended pattern area developable. After development, such a slanting wall can be found that the lift-off step turns out to be gentle due to the accessibility of the residual photoresist to the solvent.

Following the in-house CMOS-compatible process, the prototype is fabricated. It can be noticed that the pattern and alignment of each metal layer is in fair agreement with the proposed structure, and there is hardly any metal exfoliation as shown in Fig. 22(a). Measured by a Vernier caliper, the dimension of the chip is $2.8 \times 2.8 \mathrm{~mm}^{2}$. The chip side length is $0.4 \mathrm{~mm}$ longer than that in the simulation model. This extension is kept to ensure that the device is not damaged by the dicing blade when the fabricated chip is diced into smaller samples. The cross section is viewed by an optical microscope, and the realized total oxide thickness is found to be $18.18 \mu \mathrm{m}$ within a slight error of $0.18 \mu \mathrm{m}$ from the simulation model.

\section{B. Measurement}

The measurement of input impedance and radiation performance has been carried out in an Orbit $\mu$-lab anechoic chamber integrated with an Agilent vector network analyzer (VNA) 5242A and relevant extender modules. The antenna under test (AUT) is fed by an Infinity probe I110-A-GSG-150. The setup of the impedance measurement is shown in Fig. 23. Once the system has been calibrated with a Cascade 104-783 W-band impedance standard substrate (ISS), the AUT is then placed on a foam chuck at the center of the chamber. The simulated and measured reflection coefficients of the AUT are in fair agreement for in-band response, as shown in Fig. 24. It is evident that the on-chip monopole antenna is well matched from 92 to $98 \mathrm{GHz}$ with a return loss of $16 \mathrm{~dB}$ at $94 \mathrm{GHz}$.

The radiation performance has been characterized in the same chamber, where the probe-fed AUT is connected to port 1 , and the scalar-feed-horn-10 (SFH-10) reference antenna is excited by port 2, as shown in Fig. 25. Here the reference antenna is set as the transmitter and the AUT works as the receiver. Fig. 26(a) and (b) give the measured radiation pattern. Due to the physical constraints of the chamber, the E-plane measurement of the AUT ranges from $\theta=-20^{\circ}$ to $230^{\circ}$ while $\varphi$ $=-90^{\circ}$, and H-plane is $\theta=0^{\circ}$ to $360^{\circ}$ while $\varphi$ is zero. Generally, the measured curves follow the same trends as the simulated

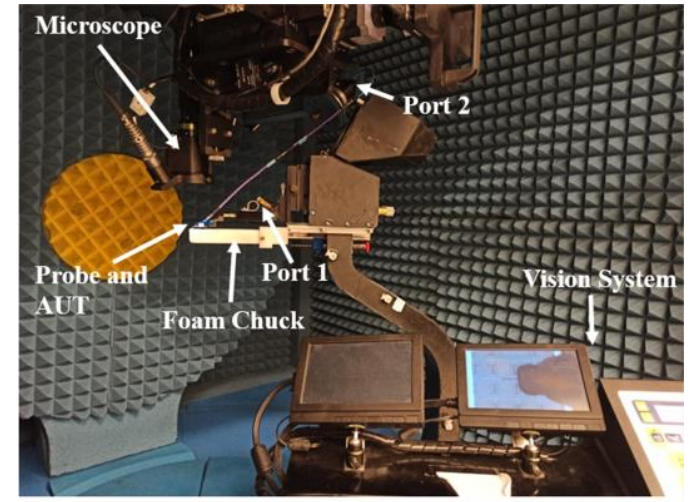

(a)
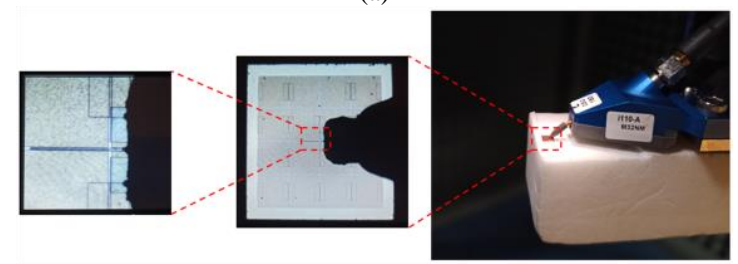

(b)

Fig. 23. Reflection coefficient measurement setup: (a) SNF anechoic chamber, (b) Probe landing.

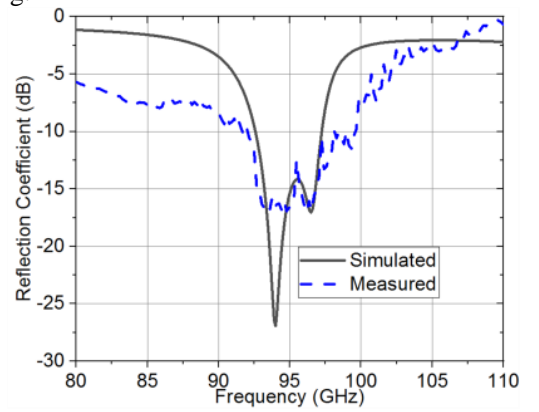

Fig. 24. Simulated and measured reflection coefficient of the AUT.

curves, however, there are some discrepancies between them. The H-plane pattern is almost matched to simulated curve except a backside radiated lobe, while there are several additional side lobes measured in the E-plane. According to Fig. 26(c), the measured peak gain is also in fair agreement with the simulated results, showing an initial rise followed by a drop, in the impedance-matched frequency band ( 92 to $98 \mathrm{GHz}$ ). The highest value of the realized gain occurs at $94 \mathrm{GHz}$ as $5.85 \mathrm{dBi}$, which is close to the gain value of $5.08 \mathrm{dBi}$ in simulations. The $3 \mathrm{~dB}$ gain bandwidth is $5.4 \%$. Furthermore, to characterize the directivity $D$ and radiation efficiency $\eta$ of the prototype, the spherical radiation pattern with the exception of the probe part $\left(\varphi=-30^{\circ}\right.$ to $+30^{\circ}, \theta=20^{\circ}$ to $\left.130^{\circ}\right)$ has been measured with an azimuth step of $5^{\circ}$ and the inclination step of $2^{\circ}$. The measured data is substituted into the following equations:

$$
\begin{gathered}
D=\frac{\sum_{\varphi, \theta} \sin \theta}{\sum_{\varphi, \theta} F_{n}(\varphi, \theta) \sin \theta} \\
\eta=\frac{G}{D}
\end{gathered}
$$

where $F_{n}(\varphi, \theta)$ is the normalized radiation pattern. Then the directivity is $8.22 \mathrm{~dB}$ and the radiation efficiency is $57 \%$. 


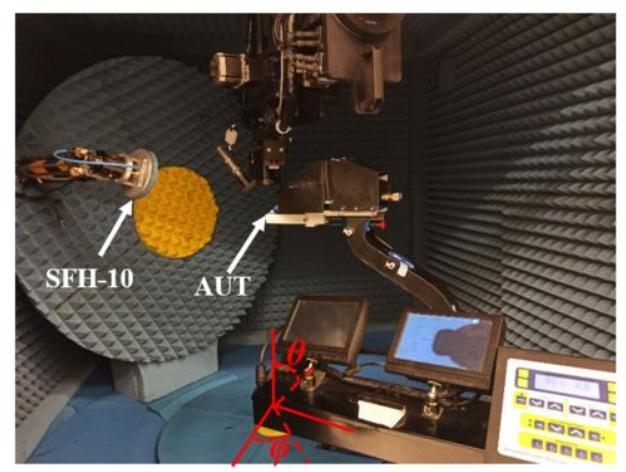

Fig. 25. Radiation measurement setup.

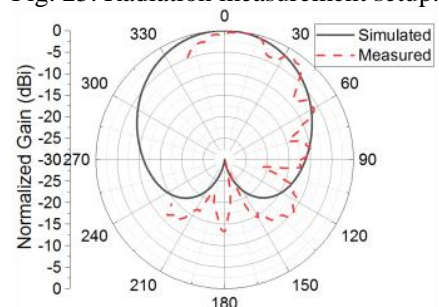

(a)

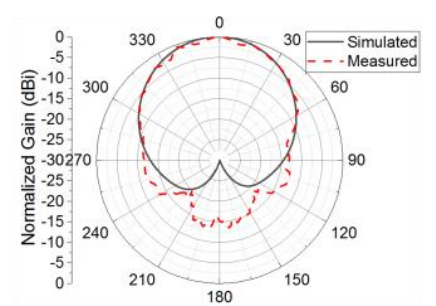

(b)

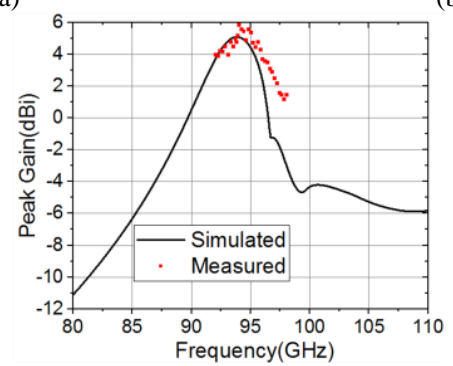

(c)

Fig. 26. Simulated and measured: (a) Normalized radiation patterns at $94 \mathrm{GHz}$ in E-plane and (b) H-plane. (c) Peak gain versus frequency.

\section{Discussion}

It is anticipated that the discrepancy in impedance characterization, radiation pattern and gain is caused by the extra chip area and probe effect. Due to the risks associated with dicing, the side length of each sample is expanded to 2.8 $\mathrm{mm}$ instead of $2.4 \mathrm{~mm}$, so the ground plane has also expanded with the increased chip size. Secondly, the huge conductor body and the uncovered tips of the feeding probe near the AUT bring unwanted coupling, self-radiation and reflection. Fig. 27(a) shows the post-measurement simulation model where the chip margin and probe body are both taken into consideration.

The post-simulation results show side lobes and back lobe in radiation pattern that are in decent agreement with the measured results. The matched reflection coefficient and the curve of gain versus frequency also verify that the main reasons for the discrepancy are the extra chip margin and the huge conductive feeding probe. It is envisioned that a customized absorber covering the probe and the de-embedding of the probe influence from regularly tested results would be helpful for a more accurate AoC measurement.

Table IV shows the gain-enhancement performance comparison between our work and similar works using AMC for AoC gain enhancement. According to author's best knowledge, this article presents the thinnest on-chip AMC design and the highest experimentally measured gain of an

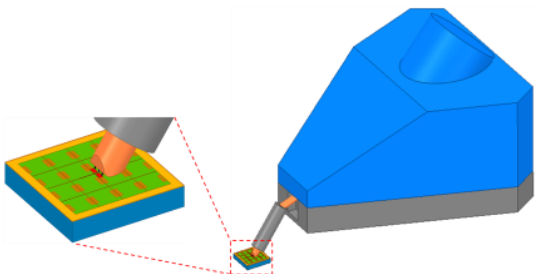

(a)
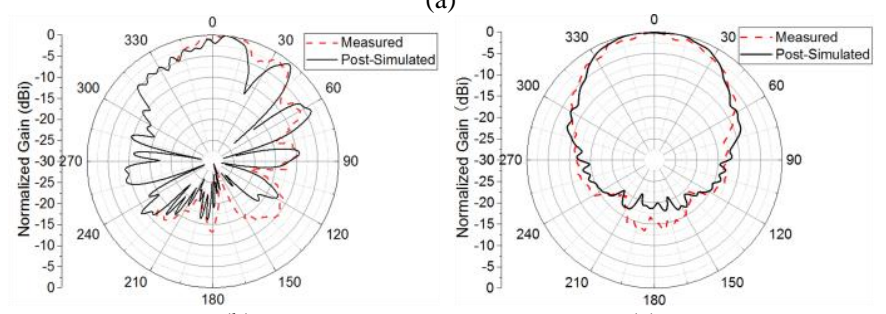

(b)

(c)

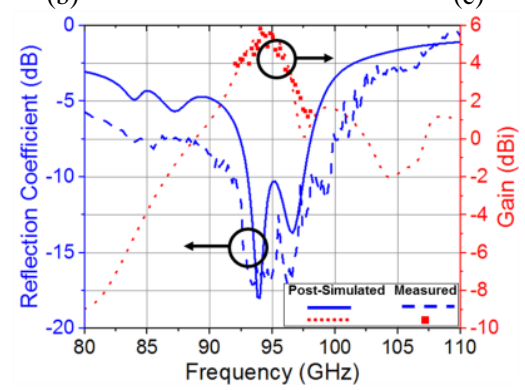

(d)

Fig. 27. Post-measurement discussion: (a) AUT with the probe model, (b) Radiation pattern in E-plane and (c) H-plane, (d) $\mathrm{S}_{11}$ and gain versus frequency.

TABLE IV

COMPARISON WITH PREVIOUSLY PUBlished AMC-BACKed AoC

\begin{tabular}{ccccccc}
\hline \hline Techniques & $\begin{array}{c}t_{A M C} \\
(\mu \mathrm{m})\end{array}$ & $\begin{array}{c}\text { Measured } \\
\text { Gain }(\mathrm{dBi})\end{array}$ & $\begin{array}{c}\text { Incr. in } \\
\text { Gain }(\mathrm{dB})\end{array}$ & $\eta$ & $\begin{array}{c}\text { Incr. in } \\
\eta\end{array}$ \\
\hline$[41]$ & $\begin{array}{c}\text { Ground plane } \\
\text { underneath Si }\end{array}$ & 300 & 0.12 & $\mathrm{NR}$ & $\mathrm{NR}$ & $\mathrm{NR}$ \\
\hline$[38] \quad \begin{array}{c}\text { Two PRS layers } \\
+ \text { Ground plane } \\
\text { underneath Si }\end{array}$ & 250 & 0 & 3.3 & $\begin{array}{c}21 \% \\
(\mathrm{sim})\end{array}$ & $\begin{array}{c}10 \% \\
(\mathrm{sim})\end{array}$ \\
\hline $\begin{array}{c}\text { Slotted patch + } \\
\text { Ground plane } \\
\text { above Si }\end{array}$ & 20 & -1.4 & 8 & $\mathrm{NR}$ & $\mathrm{NR}$ \\
\hline This & $\begin{array}{c}\text { EGS + Ground } \\
\text { plane above Si + } \\
\text { No adhesion } \\
\text { layers }\end{array}$ & 16 & 5.85 & 9.15 & $57 \%$ & $42 \%$ \\
\hline \hline
\end{tabular}

antenna by using on-chip AMC only.

\section{CONCLUSION}

Although SoC has become a promising solution to realize the modern wireless systems on a chip, the silicon substrate is unsuitable for hosting antennas on it. AMC implemented on a chip, whose ground plane is located on top of the substrate, cannot only isolate the lossy Si substrate, also provide in-phase reflection for the AoC, thereby alleviating the crosstalk between the antenna and on-chip circuits and enhancing the gain. However, the required AMC thickness is not compatible with typical CMOS processes. To reduce AMC thickness, two kinds of thickness miniaturization techniques, MP and EGS, have been demonstrated in this article. Smartly using the vias and metal layers available in the CMOS stack-up, an ultra-thin 
$94 \mathrm{GHz}$ AMC is realized with a comparable thickness $(16 \mu \mathrm{m})$ with the oxide layer of typical CMOS stack-up. Moreover, the conventional CMOS stack-up with low-conductivity adhesion layers brings some undesired losses. This article presents an in-house CMOS-compatible process. The adhesion of the metallic layer to the oxide has been improved without using adhesion layers. The measured input impedance and radiation performance of the AoC backed by the proposed ultra-thin AMC is fairly consistent with the simulations. It provides 5.85 $\mathrm{dBi}$ gain with a return loss of $16 \mathrm{~dB}$ at $94 \mathrm{GHz}$.

\section{REFERENCES}

[1] H. M. Cheema, F. Khalid, and A. Shamim, Antenna-on-Chip: Design, Challenges, and Opportunities. Boston, MA, USA: Artech House, 2021.

[2] R. Karim and P. Malcovati, "On-Chip-Antennas: Next Milestone in the Big World of Small Satellites-A Survey of Potentials, Challenges, and Future Directions," IEEE Aerospace and Electronic Systems Magazine, vol. 36, no. 1, pp. 46-60, 2021, doi: 10.1109/MAES.2020.3016751.

[3] S. Mandal, S. K. Mandal, R. Mahapatra, and A. K. Mal, "Studies on on-chip antenna using standard CMOS technology," in 2017 Devices for Integrated Circuit (DevIC), 23-24 March 2017 2017, pp. 471-475, doi: 10.1109/DEVIC.2017.8073994.

[4] H. M. Cheema and A. Shamim, "The last barrier: on-chip antennas," IEEE Microwave Magazine, vol. 14, no. 1, pp. 79-91, 2013, doi: $10.1109 / \mathrm{mmm} .2012 .2226542$.

[5] R. Karim, A. Iftikhar, and R. Ramzan, "Performance-Issues-Mitigation-Techniques for On-Chip-Antennas - Recent Developments in RF, MM-Wave, and Thz Bands With Future Directions," IEEE Access, vol. 8, pp. 219577-219610, 2020, doi: 10.1109/ACCESS.2020.3042928.

[6] R. Karim, A. Iftikhar, B. Ijaz, and I. Ben Mabrouk, "The Potentials, Challenges, and Future Directions of On-Chip-Antennas for Emerging Wireless Applications-A Comprehensive Survey," IEEE Access, vol. 7, pp. 173897-173934, 2019, doi: 10.1109/access.2019.2957073.

[7] U. Johannsen and A. B. Smolders, "Antenna-on-Chip Integration in Mainstream Silicon Semiconductor Technologies," IET Conference Proceedings, pp. 580 (5 pp.)-580 (5 pp.). [Online]. Available: https://digital-library.theiet.org/content/conferences/10.1049/cp.20 $\underline{18.0939}$

[8] A. Babakhani, X. Guan, A. Komijani, A. Natarajan, and A. Hajimiri, "A 77-GHz phased-array transceiver with on-chip antennas in silicon: Receiver and antennas," IEEE Journal of Solid-State Circuits, vol. 41, no. 12, pp. 2795-2806, 2006.

[9] S. Mandal, S. K. Mandal, and A. K. Mal, "On-chip antennas using standard CMOS technology: A brief overview," in 2017 International Conference on Innovations in Electronics, Signal Processing and Communication (IESC), 6-7 April 2017 2017, pp. 74-78, doi: 10.1109/IESPC.2017.8071868.

[10] H. J. Ng, R. Wang, and D. Kissinger, "On-Chip Antennas in SiGe BiCMOS Technology: Challenges, State of the Art and Future Directions," in 2018 Asia-Pacific Microwave Conference (APMC), 6-9 Nov. 2018 2018, pp. 621-623, doi: 10.23919/APMC.2018.8617626.

[11] A. Shamim and H. Zhang, "On-chip antenna: challenges and design considerations," in Telecommunications, Antennas and Propagation for $5 G$ and Beyond: Institution of Engineering and Technology, 2020, pp. 123-155. [Online]. Available: https://digital-library.theiet.org/content/books/10.1049/pbte093e_c $\underline{\mathrm{h} 6}$

[12] F. A. Ghaffar, M. U. Khalid, K. N. Salama, and A. Shamim, "24-GHz LTCC fractal antenna array SoP with integrated Fresnel lens," IEEE Antennas and Wireless Propagation Letters, vol. 10, pp. 705-708, 2011.

[13] J. Grzyb, M. Andree, R. Jain, B. Heinemann, and U. R. Pfeiffer, "A Lens-Coupled On-Chip Antenna for Dual-Polarization SiGe HBT THz Direct Detector," IEEE Antennas and Wireless Propagation Letters, vol. 18, no. 11, pp. 2404-2408, 2019, doi: 10.1109/LAWP.2019.2927300.
J. Grzyb, K. Statnikov, N. Sarmah, and U. R. Pfeiffer, "A wideband $240 \mathrm{GHz}$ lens-integrated circularly polarized on-chip annular slot antenna for a FMCW radar transceiver module in SiGe technology," in 2015 SBMO/IEEE MTT-S International Microwave and Optoelectronics Conference (IMOC), 3-6 Nov. 2015 2015, pp. 1-4, doi: 10.1109/IMOC.2015.7369165.

[15] R. Hahnel, B. Klein, and D. Plettemeier, "Integrated pseudo-lens structures for on-chip antennas at $180 \mathrm{GHz}, "$ in 2016 International Symposium on Antennas and Propagation (ISAP), 24-28 Oct. 2016 2016, pp. 784-785.

[16] K. Statnikov, N. Sarmah, J. Grzyb, S. Malz, B. Heinemann, and U. R. Pfeiffer, "A $240 \mathrm{GHz}$ circular polarized FMCW radar based on a SiGe transceiver with a lens-integrated on-chip antenna," in 2014 44th European Microwave Conference, 6-9 Oct. 2014 2014, pp. 1750-1753, doi: 10.1109/EuMC.2014.6986795.

[17] W. H. Syed, G. Fiorentino, D. Cavallo, M. Spirito, P. M. Sarro, and A. Neto, "Design, fabrication, and measurements of a $0.3 \mathrm{THz}$ on-chip double slot antenna enhanced by artificial dielectrics," IEEE Transactions on Terahertz Science and Technology, vol. 5, no. 2, pp. 288-298, 2015.

[18] H. Zhang and A. Shamim, "Gain enhancement of millimeter-wave on-chip antenna through an additively manufactured functional package," IEEE Transactions on Antennas and Propagation, vol. 68, no. 6, pp. 4344-4353, 2020.

[19] J. Grzyb, L. Duxian, U. Pfeiffer, and B. Gaucher, "Wideband Cavity-backed Folded Dipole Superstrate Antenna for $60 \mathrm{GHz}$ Applications," in 2006 IEEE Antennas and Propagation Society International Symposium, 9-14 July 2006 2006, pp. 3939-3942, doi: 10.1109/APS.2006.1711487.

[20] J. Xu and D. S. Ricketts, "Broadband W-band on-chip Yagi antenna using superstrate for high efficiency and endfire radiation," in 2014 Asia-Pacific Microwave Conference, 4-7 Nov. 2014 2014, pp. 360-362.

[21] M. Seyyed-Esfahlan, M. Kaynak, B. Göttel, and I. Tekin, "SiGe process integrated on-chip dipole antenna on finite-size ground plane," IEEE Antennas and Wireless Propagation Letters, vol. 12, pp. 1260-1263, 2013.

[22] W. A. Ahmad, M. Kucharski, A. Di Serio, H. J. Ng, C. Waldschmidt, and D. Kissinger, "Planar highly efficient high-gain $165 \mathrm{GHz}$ on-chip antennas for integrated radar sensors," IEEE Antennas and Wireless Propagation Letters, vol. 18, no. 11, pp. 2429-2433, 2019.

[23] H. Chu, Y. Guo, T. Lim, Y. M. Khoo, and X. Shi, "135-GHz Micromachined On-Chip Antenna and Antenna Array," IEEE Transactions on Antennas and Propagation, vol. 60, no. 10, pp. 4582-4588, 2012, doi: 10.1109/TAP.2012.2209855.

[24] W. Ahmad, M. Kucharski, H. Ng, and D. Kissinger, "A Compact Efficient D-Band Micromachined On-Chip Differential Patch Antenna for Radar Applications*," in 2019 IEEE International Symposium on Antennas and Propagation and USNC-URSI Radio Science Meeting, 7-12 July 2019 2019, pp. 2201-2202, doi: 10.1109/APUSNCURSINRSM.2019.8889358.

[25] W. T. Khan et al., "A D-Band Micromachined End-Fire Antenna in 130-nm SiGe BiCMOS Technology," IEEE Transactions on Antennas and Propagation, vol. 63, no. 6, pp. 2449-2459, 2015, doi: 10.1109/TAP.2015.2416751.

[26] H. J. Ng, J. Wessel, D. Genschow, R. Wang, Y. Sun, and D. Kissinger, "Miniaturized $122 \mathrm{GHz}$ system-on-chip radar sensor with on-chip antennas utilizing a novel antenna design approach," in 2016 IEEE MTT-S International Microwave Symposium (IMS), 22-27 May 2016 2016, pp. 1-4, doi: 10.1109/MWSYM.2016.7540353.

[27] K. T. Chan et al., "Integrated antennas on Si with over $100 \mathrm{GHz}$ performance, fabricated using an optimized proton implantation process," IEEE Microwave and Wireless Components Letters, vol. 13, no. 11, pp. 487-489, 2003, doi: 10.1109/lmwc.2003.817146.

[28] T. Hirano et al., "Design of $60 \mathrm{GHz}$ CMOS on-chip dipole antenna with $50 \%$ radiation efficiency by helium-3 ion irradiation," in 2015 IEEE Conference on Antenna Measurements \& Applications (CAMA), 30 Nov.-2 Dec. 2015 2015, pp. 1-2, doi: 10.1109/CAMA.2015.7428176.

[29] R. Wu et al., "A 60-GHz efficiency-enhanced on-chip dipole antenna using helium-3 ion implantation process," in 2014 44th European Microwave Conference, 6-9 Oct. 2014 2014, pp. 108-111, doi: 10.1109/EuMC.2014.6986381. 
J. Sato and T. Murata, "140 GHz CMOS on-chip dipole antenna with optimal ion-irradiated-silicon with vertical reflector," in 2015 International Symposium on Antennas and Propagation (ISAP), 9-12 Nov. 2015 2015, pp. 1-3.

[31] M. Nafe, A. Syed, and A. Shamim, "Gain-enhanced on-chip folded dipole antenna utilizing artificial magnetic conductor at $94 \mathrm{GHz}, "$ IEEE Antennas and Wireless Propagation Letters, vol. 16, pp. 2844-2847, 2017.

[32] O. Markish, O. Katz, B. Sheinman, D. Corcos, and D. Elad, "On-Chip Millimeter Wave Antennas and Transceivers," presented at the Proceedings of the 9th International Symposium on Networks-on-Chip, Vancouver, BC, Canada, 2015. [Online]. Available: https://doi.org/10.1145/2786572.2789983.

[33] Y. Yu, Z. Akhter, and A. Shamim, "Ultra- Thin Artificial Magnetic Conductor with Metallic Posts for a $94 \mathrm{GHz}$ On-chip Antenna," in 2021 International Applied Computational Electromagnetics Society Symposium (ACES), 1-5 Aug. 2021 2021, pp. 1-3, doi: 10.1109/ACES53325.2021.00181.

[34] C. A. Balanis, Antenna theory: analysis and design. John wiley \& sons, 2015.

[35] B. S. Cook and A. Shamim, "Utilizing Wideband AMC Structures for High-Gain Inkjet-Printed Antennas on Lossy Paper Substrate," IEEE Antennas and Wireless Propagation Letters, vol. 12, pp. 76-79, 2013, doi: 10.1109/LAWP.2013.2240251.

[36] H. Mosallaei and K. Sarabandi, "Antenna Miniaturization and Bandwidth Enhancement Using a Reactive Impedance Substrate,"

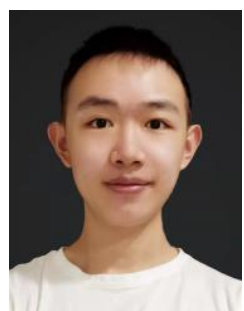

Yiyang Yu received his B.Eng. degree in electromagnetics and wireless technology from University of Electronic Science and Technology of China (UESTC), China, in 2019, and the M.S. degree in electrical and computer engineering from King Abdullah University of Science and Technology (KAUST), Saudi Arabia, in 2021, where he is currently pursuing the Ph.D. degree.

His current research interests include millimeter-wave antennas, on-chip antennas, reflect-array antennas, and electrically small antennas.

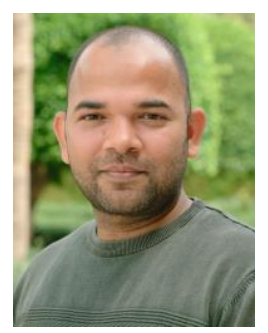

Zubair Akhter received a bachelor's degree in electronics and instrumentation engineering from the Anand Engineering College, Agra, India, in 2008, and the M.Tech. Degree in RF and microwave engineering from Indian Institute of Technology Roorkee, Roorkee, India, in 2011. He obtained his Ph.D. in Electrical Engineering from the Indian Institute of Technology, Kanpur, India, in 2018, with a focus on microwave imaging, material characterization, non-destructive testing of materials, through-wall imaging, and ultra-wide-band antennas for imaging applications. He is currently associated with King Abdullah University of Science and Technology (KAUST), Saudi Arabia as a Post-doctoral fellow. His current research interests are in printed-flexible electronics, printed transparent antennas, chipless RFID sensing, antennas for IoT applications, ultra-thin UAV antennas, microwave sensing for oil and gas industry, Imaging, and Non-destructive Testing. He
IEEE Transactions on Antennas and Propagation, vol. 52, no. 9, pp. 2403-2414, 2004, doi: 10.1109/tap.2004.834135.

[37] J. Volakis, C. C. Chen, and K. Fujimoto, Small Antennas:Miniaturization Techniques \& Applications. McGraw-Hill Education, 2009.

[38] Q. Liu, A. J. v. d. Biggelaar, U. Johannsen, M. C. v. Beurden, and A. B. Smolders, "On-Chip Metal Tiling for Improving Grounded mm-Wave Antenna-on-Chip Performance in Standard Low-Cost Packaging," IEEE Transactions on Antennas and Propagation, vol. 68, no. 4, pp. 2638-2645, 2020, doi: 10.1109/TAP.2019.2957713.

[39] L. Marnat, A. A. A. Carreno, D. Conchouso, M. G. Martinez, I. G. Foulds, and A. Shamim, "New Movable Plate for Efficient Millimeter Wave Vertical on-Chip Antenna," IEEE Transactions on Antennas and Propagation, vol. 61, no. 4, pp. 1608-1615, 2013, doi: 10.1109/tap.2013.2241720.

[40] S. Pan, C. Guclu, and F. Capolino, "Effect of losses on the performance of very thin artificial magnetic conductors," in 2013 International Symposium on Electromagnetic Theory, 2013: IEEE, pp. 404-407.

[41] C. Hui, Q. Lu, and G. Yong-Xin, "60-GHz broadband CMOS on-chip antenna with an artificial magnetic conductor," in 2016 IEEE MTT-S International Microwave Workshop Series on Advanced Materials and Processes for RF and THz Applications (IMWS-AMP), 20-22 July 2016 2016, pp. 1-2, doi: 10.1109/IMWS-AMP.2016.7588396.

has authored or co-authored over 55 scientific contributions published in peer-reviewed journals, patents and various admired international conferences. Dr. Akhter is a recipient of Early Career Researcher Teaching Award from the Office of the Provost in collaboration with Office of Vice President (Research) at King Abdullah University of Science and Technology, KAUST. Dr. Akhter is the founding Chair of the IEEE Antennas and Propagation Society Student Branch Chapter IITK, Uttar Pradesh Section, India.

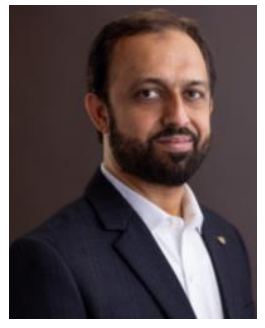

Atif Shamim received his MS and $\mathrm{PhD}$ degrees in electrical engineering from Carleton University, Canada in 2004 and 2009 respectively. He was an NSERC Alexander Graham Bell Graduate scholar at Carleton University from 2007 till 2009 and an NSERC postdoctoral Fellow in 2009-2010 at Royal Military College Canada and KAUST. In August 2010, he joined the Electrical Engineering Program at KAUST, where he is currently an Associate Professor and principal investigator of IMPACT Lab. He was an invited researcher at the VTT Micro-Modules Research Center (Oulu, Finland) in 2006. His research work has won best paper awards in IEEE IMS 2016, IEEE MECAP 2016, IEEE EuWiT 2008, first prize in IEEE IMS 2019 3MT competition and finalist/honorable mention prizes in IEEE APS Design Competition 2020, IEEE APS 2005, IEEE IMS 2014, IEEE IMS 2017 (3MT competition), R. W. P. King IEEE Award for journal papers in IEEE TAP 2017 and 2020. He was given the Ottawa Centre of Research Innovation (OCRI) Researcher of the Year Award in 2008 in Canada. His work on Wireless Dosimeter won the ITAC SMC Award at Canadian Microelectronics TEXPO in 2007. Prof. Shamim also won numerous business-related awards, including 1st prize in 
Canada's national business plan competition and was awarded OCRI Entrepreneur of the year award in 2010. He has been awarded the Kings Prize for the best innovation of the year (2018) for his work on sensors for the oil industry. He is an author of 1 Book, 3 Book Chapters and over 250 publications, an inventor on 35 patents and has given more than 70 invited talks at various international forums. His research interests are in innovative antenna designs and their integration strategies with circuits and sensors for flexible and wearable wireless sensing systems through a combination of CMOS and additive manufacturing technologies. He has been selected as IEEE AP-S Distinguished Lecturer for the period 2022-2024. He is the Senior Member of IEEE, Member of IEEE APS Measurements Committee and IEEE MTT Microwave Control Techniques Committee, founded the first IEEE AP/MTT chapter in Saudi Arabia (2013) and served on the editorial board of IEEE Transactions on Antennas and Propagation (2013-2019), and as a Guest Editor for IEEE AWPL Special issue (2019), and is currently serving as an Associate Editor for IEEE Journal of Electromagnetics, RF and Microwaves in Medicine and Biology. 